\title{
Determination of Antiaflatoxigenic Effect of Probiotic Strains in Sorghum bicolour
}

\author{
P.B. Padmaja and S. Periyar Selvam* \\ Department of Food Process Engineering, SRM University, Kattankulathur - 603203, India. \\ http://dx.doi.org/10.13005/bbra/2138
}

(Received: 09 March 2016; accepted: 19 April 2016)

\begin{abstract}
Sorghum is a food and feed crop grown commonly in Asian and African countries. Aflatoxin contamination of sorghum is a common problem in all tropical countries. Since even low doses of aflatoxin intake, over a period of time, can prove to be carcinogenic, removal of aflatoxin from sorghum is necessary. Biodecontamination of food grains is now gaining popularity since it is considered to be a safer alternative to chemical methods. Probiotic organisms such as lactic acid bacteria (LAB), have been commonly used in fermentation of various food products. Studies have been done to prove the ability of the probiotic organisms to bind and remove aflatoxin from food. Here the ability of four strains of lactic acid bacteria (Lactobacillus plantarum, Lactobacillus rhamnosus, Lactobacillus brevis, and Lactobacillus delbrueckii subsp. lactis) to reduce aflatoxin levels in sorghum during fermentation has been studied. The initial and final aflatoxin content in sorghum was analyzed using HPLC and UV spectrophotometer. It was concluded that $\mathrm{L}$. delbrueckii had the maximum antiaflatoxigenic ability. It showed $\mathbf{7 4 . 3 8 \%}$ reduction of aflatoxin in Sorghum bicolor. The research outcome concluded that L. delbrueckii may be used as potential probiotic for reducing the aflatoxin content in sorghum.
\end{abstract}

Key words: Sorghum bicolor, probiotics, aflatoxin, HPLC, TLC.

The tropical climate, post harvest storage conditions and agricultural practices of countries such as India, Brazil, Nigeria, etc. creates an ideal condition for fungal growth. When these fungi infect the crops, they secrete a toxic secondary metabolite making the crop unsafe for animal and human consumption ${ }^{1-4}$. These secondary metabolites or mycotoxins lead to loss in yield, nutritive losses and detoriation of health in human beings and animals. Though there are a large number of mycotoxins, the ones that cause major economic loss in food crops are aflatoxins, ochratoxin, fumonisins and patulin. These are

\footnotetext{
* To whom all correspondence should be addressed. Mob.: +91-9444821490;

E-mail: periyar.india@gmail.com
}

produced by mould belonging to the genus Aspergillus, Penicillium and Fusarium respectively ${ }^{5,6,7}$.

The aflatoxins are classified into four main types based on the colour they fluoresce under ultraviolet (UV) light. They are aflatoxin B1 (AFB1), aflatoxin B2 AFB2), aflatoxin G1 (AFG1) and aflatoxin G2 (AFG2) ${ }^{8}$. Out of these, AFB1 is considered to be the most potent mycotoxin since it causes mycotoxicosis and hepatic cancer in humans and animals with even low levels of intake, ${ }^{10}$. Though good agricultural and storage practices are the best way to prevent fungal contamination of food crops, farmers in developing countries such as India are not always able to keep up the required standards of cultivation and storage that prevents fungal infestation of the crops. This leads to fungal 
growth and toxin production over the crops making them unfit for human consumption.

Sorghum bicolor is a crop widely grown in the African and Asian countries. Initially it was used only as animal feed, but now it is gaining popularity as a food crop for human use owing to its drought resistant nature hence, helping in eradicating food shortage. The annual growth in sorghum production in Asian and African countries has been remarkable. It has become the staple cereal for over 750 million individuals in these continents $^{11-13}$. It is majorly cultivated in the Africa, Asia and the Americas ${ }^{14}$. It is commonly used to make porridge and other baked products such as bread, cakes and cookies ${ }^{15}$.

Though sorghum has intrinsic defence systems to prevent pest and microbe infestations, the innate systems are unable to prevent contamination by fungi which in turn secrete mycotoxin hence making the food crop unsafe for human consumption ${ }^{12,16}$. The annual economic loss due to fungal and mycotoxin contamination of sorghum has been estimated to be over 130 million USD. Aflatoxin has been found to be the major source of contamination in Sorghum bicolor causing birth defects and stunted growth in children apart from being the foremost causative agent of hepatic tumours in African and Asian countries $^{13}$. The rising importance of sorghum as a staple food makes it necessary to come up with effective ways to decontaminate it. Though physical and chemical methods of aflatoxin decontamination are available, biological methods are usually preferred since they bring about least damage to the crops' nutritive value ${ }^{17}$.

Fermentation of sorghum has been a common age old practice in African and Asian countries. It has been found that fermentation improves the nutritional and digestive properties of sorghum. Also in recent times, with the growing awareness about food allergies, the need for healthy gluten free products is growing. Fermentation of sorghum yields flour that can be used to improve the texture and nutritional content of gluten free foods such as rice pasta ${ }^{18,19,20}$.

Probiotic bacteria such as lactic acid bacteria (LAB), Bifidobacteria, Propionibacterium spp. have been found to reduce toxin concentration in solution ${ }^{21}$. The bacteria noncovalently bind the toxin to their cell wall and hence remove it from liquid media. Since LAB have been declared as Generally Regarded As Safe (GRAS) organisms by Food and Agriculture Organisation (FAO), they are ideally suited to be used as starter cultures during sorghum fermentation ${ }^{21,22}$. Apart from in improving its texture and nutritional content, it also helps in reducing the aflatoxin content of sorghum hence making it safe for consumption.

In this study, we have screened the ability of four lactic acid bacteria in reducing the aflatoxin content of sorghum. The aim of the study is to identify the LAB strain with maximal antiaflatoxigenic ability.

\section{MATERIALSAND METHODS}

\section{Culture revival}

The starter cultures to be used for fermentation were obtained from MTCC. All four cultures (Lactobacillus plantarum (MTCC no. 4464), Lactobacillus rhamnosus (MTCC no. 1423), Lactobacillus brevis (MTCC no. 4463), and Lactobacillus delbrueckii subsp. lactis (MTCC no. 911) were received as lyophilized powder. They were revived by incubating in de Mann Rogosa Sharpe (MRS) broth at $34^{\circ} \mathrm{C}$ for 24 hours after which the culture was streaked onto MRS agar plates and incubated at $34^{\circ} \mathrm{C}$ for 48 hours. The colonies that grew in the plates were further subcultured and used for fermentation of sorghum.

\section{Fermentation of Sorghum}

Sorghum grains used were obtained from the local super market. Bacterial culture suspensions to be used as starter cultures for fermentation was prepared by addition of the bacterial colonies, from overnight culture agar plates , to a sodium chloride suspension $(0.9 \% \mathrm{w} /$ v). Sorghum samples were prepared for fermentation by mixing the grains with distilled water $(1: 10, \mathrm{w} / \mathrm{v})$ and sterilizing it prior to inoculation with the starter culture suspensions $(5 \%, \mathrm{w} / \mathrm{v})$. All samples were inoculated in triplicates and incubated at $35^{\circ}$ for 5 days in a shaker incubator to enable constant agitation ${ }^{23}$.

\section{Extraction of Aflatoxin}

Once fermentation was complete, the grains were separated from the water to perform aflatoxin analysis. To $5 \mathrm{~g}$ of the fermented sample, $20 \mathrm{ml}$ of $80 \%$ methanol containing $1 \mathrm{~g}$ sodium 
chloride was added. This was allowed to mix well by placing in a magnetic stirrer. The sample was defatted by adding $20 \mathrm{ml}$ hexane and the hexane fraction was eliminated in a separating funnel. The final methanolic extract was filtered using Whatmann no. 1filter paper and the solvent evaporated in a water bath. The residue present was redissolved in $2 \mathrm{ml}$ of $50 \%$ methanol $^{24}$.

Analysis of aflatoxin content by thin layer chromatograghy and UV spectrophotometer

The methanolic extract was quantitatively analyzed using TLC and UV spectrophotometer to determine the amount of aflatoxin present in sorghum before and after fermentation. In a precoated silica gel TLC plate, the extracts were spotted and the plate was developed in the TLC tank containing the solvent system chloroform: acetone $(6: 4, \mathrm{v} / \mathrm{v})$. Aflatoxin standards obtained from Sigma-Aldrich was spotted alongside the samples for comparison. The aflatoxin presence was detected by viewing the plates under UV light of 365nm wavelength. The spots that fluoresced blue under UV light were scrapped of and dissolved in $5 \mathrm{ml}$ ice cold methanol by centrifuging at 5000rpm for 5 minutes. To quantify the amount of aflatoxin present, absorbance of the supernatant obtained was found by means of a UV spectrophotometer (Jasco V-730) at $365 \mathrm{~nm}^{25}$ and substituting the value in the equation:

AFB1 content $(\mathrm{ig} / \mathrm{ml})=(\mathrm{D} \times 312) /(21800 \times \mathrm{l}) \times 1000$

Where,

$\mathrm{D}$ is the absorbance

$l$ is the path length ( $1 \mathrm{~cm}$ cuvette was used)

312 is the molecular weight (M) of AFB1 and 21800 is the extinction coefficient $\left(\sum\right)$

Analysis of Aflatoxin Content by High Performance Liquid Chromatography (HPLC)

The methanolic extract was analyzed using HPLC to accurately quantify the aflatoxin content. $25 \mu \mathrm{l}$ of the sample was injected into an Agilent 1260 Infinity HPLC instrument with Zorba eclipse plus C18 $5 \mu \mathrm{m}$ column and detected using a fluorescence detector at 365nm excitation and $460 \mathrm{~nm}$ emission. The mobile phase was a mixture of acetonitrile, methanol and water in the ratio of 25:25:50 (v/v/v). The aflatoxin peak was identified by comparing with standard peaks and quantified using the peak area obtained ${ }^{17}$.

\section{Statistical analysis}

The data obtained was analyzed by
ANNOVA using SPSS/12.0 software at $\mathrm{p}<0.05$ to conclude if there was a significant difference between the values obtained.

\section{RESULTSAND DISCUSSION}

In this study we compared the abilities of four strains of LAB in reducing aflatoxin content of Sorghum bicolour during fermentation. The property of LAB that makes them bind and remove aflatoxin B1 from solution has already been established in a number of studies. Through this work we identified the LAB strain with maximal antiaflatoxigenic activity, during fermentation of sorghum, out of four different strainsLactobacillus plantarum, Lactobacillus delbrueckii subsp. lactis, Lactobacillus brevis and Lactobacillus plantarum. Preliminary reduction was studied through TLC and UV spectrophotometer and later the results were confirmed using HPLC. Though TLC-UV spectrophotometric method of quantitatively analysing the AFB1 content is still in use today, other methods such as HPLC are being preferred since the former method has lower sensitivity, accuracy and sample recovery values ${ }^{26,27}$. The reduction potentials of the $\mathrm{LAB}$ were analysed using HPLC in order to compare the precision of HPLC and TLC.

To compare the efficacy of these two methods of analysis, the values obtained were statistically analysed. It was found that the values of percentage reduction obtained through each of the methods were not significantly different. This shows that the recovery potentials of both the chromatographic methods are equally good. This is in agreement with the results reported by Chang et al., and Haghighi et al., ${ }^{28,29}$.

The initial concentration of aflatoxin, inherently present in the sorghum sample, was found to be $0.32 \mu \mathrm{g} / \mathrm{ml}$.

\section{Analysis of AFB1 Content in Sorghum (Post Fermentation) by Thin Layer Chromatography (TLC) and UV Spectrophotometer}

The results of the preliminary analysis, performed by TLC followed by UV spectrophotometer are presented in table I. The aflatoxin was extracted after fermentation, spotted onto TLC plates alongside AFB1 standards. $L$. delbrueckii subsp. lactis was found to have 
maximal reduction potential at $74.38 \%$. This value was significantly $(\mathrm{p}<0.05)$ higher than the reduction potential of the other three strains that were used in the study.

\section{Analysis of AFB1 content in sorghum (post fermentation) by HPLC}

Results of the quantitative estimation of AFB1 content in sorghum using HPLC is represented in table II. L. delbrueckii is found to be having the maximum reduction potential, hence confirming the results obtained through the TLCUV analysis.

Due to lack of popularity as a human feed, in vivo studies of sorghum detoxification using probiotic bacteria are very less. Recently a study conducted in Pakistan concluded that almost 50\% of the sorghum in the market contained higher than the permissible amount of AFB $1^{24}$. The increasing demand for sorghum necessitates the need to come up with detoxification procedures to make it safe for consumption.

Even though a number of physical and chemical methods are available to remove aflatoxin from sorghum, they are either too expensive or create yet another health hazard or nutrition depletion and so are not suitable to be commercially used to reduce AFB1 concentration in food crops $^{30}$. Biological methods such as fermentation using probiotic bacteria having antiaflatoxigenic activity are preferred since they are easily available, affordable, do not cause undesirable changes in the crop and in fact increase the nutritional composition by contributing to gut health of the consumer.

In this study we found that $L$. delbrueckii had the highest antiaflatoxigenic activity followed by L. rhamnosus and L. plantarum. L. brevis was found to have the least antiaflatoxgenic activity out of the four LAB that were screened. The antiaflatoxigenic activity exhibited by the bacterial species is due to the presence of techoic acid in their cell walls that bind to the aflatoxin and remove it from the medium hence making the food safe for consumption $^{31,32}$. The variation in level of antiaflatoxigenic ability of the strains is due to the difference in the components of cell walls of different strains ${ }^{32}$.

Table 1. Percentage reduction of AFB1 in Sorghum bicolor (TLC-UV)

\begin{tabular}{lll}
\hline S. No. & Organism & Percentage aflatoxin reduction (\%) \\
\hline 1 & L. brevis & $22.08 \pm 0.98^{\mathrm{a}}$ \\
2 & L. plantarum & $26.98 \pm 5.38^{\mathrm{a}}$ \\
3 & L. rhamnosus & $73.89 \pm 5.70^{\mathrm{b}}$ \\
4 & L. delbrueckii subsp. lactis & $74.38 \pm 2.96^{\mathrm{b}}$ \\
\hline
\end{tabular}

Values are representatives of mean \pm S.D. $(n=3)$. Values followed by different superscripts in a column are significantly different $(\mathrm{P}<0.05)$

Table 2. Percentage reduction of AFB1 in Sorghum bicolor (HPLC)

\begin{tabular}{lll}
\hline S. & Organism & $\begin{array}{l}\text { Percentage } \\
\text { Reduction (\%) }\end{array}$ \\
\hline 1 & L. delbrueckii subsp. lactis & $79.50 \pm 1.47^{\mathrm{a}}$ \\
2 & L. rhamnosus & $69.80 \pm 2.28^{\mathrm{b}}$ \\
3 & L. plantarum & $53.02 \pm 5.89^{\mathrm{c}}$ \\
4 & L. brevis & $48.94 \pm 0.64^{\mathrm{c}}$ \\
\hline
\end{tabular}

Values are representatives of mean \pm S.D. $(n=3)$. Values followed by different superscripts in a column are significantly different $(\mathrm{P}<0.05)$.

\section{CONCLUSION}

Out of the four LAB strains were screened for their ability to detoxify sorghum during fermentation, $L$. delbrueckii was found to have the highest detoxifying ability. Hence this is a good candidate to be used as starter culture for fermentation of Sorghum bicolor since it is found to effectively ferment sorghum as well as reduce the aflatoxin content hence increasing its nutritional quality, texture and also making it safe for consumption. This study must be used as a basis to produce these starter culture strains with 
anti-aflatoxigenic activity on a large scale to facilitate its use on a commercial basis to produce detoxified, safe and healthy fermented products of sorghum. Further work must be carried out to evaluate ways to make the cultures more sturdy and viable to enable easy usage from grassroot levels.

\section{ACKNOWLEDGMENTS}

We express our thanks to Prof. C. Muthamizchelvan, Director, Engineering Technology and Dr. M. Vairamani, Dean School of Bioengineering, SRM University and Dr Athmaselvi, Head of the department, Department of Food Process Engineering, for their help and encouragement.

\section{REFERENCES}

1. Kumar V, Basu MS, Rajendran TP. Mycotoxin research and mycoflora in some commercially important agricultural commodities. Crop Protection. 2008; 27(6): 891-905.

2. Nida'M S, Ahmad R. Mycotoxins in food from Jordan: preliminary survey. Food Control. 2010; 21(8): 1099-103.

3. Wagacha JM, Muthomi JW. Mycotoxin problem in Africa: current status, implications to food safety and health and possible management strategies. International journal of food microbiology. 2008;124(1):1-2.

4. El-Nagerabi SA, Elshafie AE, AlKhanjari SS, AlBahry SN, Elamin MR. Biological activities of Boswellia sacra extracts on the growth and aflatoxins secretion of two aflatoxigenic species of Aspergillus species. Food control. 2013; 34(2): 763-9.

5. Reddy KR, Salleh B, Saad B, Abbas HK, Abel CA, Shier WT. An overview of mycotoxin contamination in foods and its implications for human health. Toxin reviews. 2010; 29(1):3-26.

6. Chassy BM. Food safety risks and consumer health. New Biotechnology. 2010; 27(5): 53444.

7. Juodeikiene G, Bartkiene E, Basinskiene L, Matusevicius P. Mycotoxin decontamination aspects in food, feed and renewables using fermentation processes. INTECH Open Access Publisher; 2012.

8. Méndez-Albores A, Veles-Medina J, UrbinaÁlvarez E, Martínez-Bustos F, MorenoMartínez E. Effect of citric acid on aflatoxin degradation and on functional and textural properties of extruded sorghum. Animal feed science and technology. 2009;150(3): 316-29.

9. Richard JL, Payne GE, Desjardins AE, Maragos C, Norred WP, Pestka JJ. Mycotoxins: risks in plant, animal and human systems. CAST Task Force Report. 2003; 139: 101-3.

10. Jermnak U, Yoshinari T, Sugiyama Y, Tsuyuki R, Nagasawa H, Sakuda S. Isolation of methyl syringate as a specific aflatoxin production inhibitor from the essential oil of Betula alba and aflatoxin production inhibitory activities of its related compounds. International journal of food microbiology. 2012; 153(3): 339-44.

11. Faostat, F. A. O. Statistical Databases., Food and Agriculture Organization of the United Nations, 2010.

12. Ediage EN, Van Poucke C, De Saeger S. A multianalyte LC-MS/MS method for the analysis of 23 mycotoxins in different sorghum varieties: The forgotten sample matrix. Food chemistry. 2015; 177: 397-404.

13. Codex Alimentarius Commission. . Discussion paper on fungi and mycotoxins in sorghum. Joint FAO/WHO food standards programme codex committee on contaminants in foods sixth session Maastricht, The Netherlands, 26-30 March 2012.

14. Faostat, F. A. O. Statistical Databases., Food and Agriculture Organization of the United Nations, 2012.

15. US Grain Council . Sorghum. Retrieved on 19/ 12/2011 from <http://www.grains.org/Sorghum> 2010.

16. Chandrashekar A, Bandyopadhyay R, Hall AJ, Chandrashekar A, Bandyopadhyay R. Technical and institutional options for sorghum grain mold management. Proceedings of an international consultation, ICRISAT, Patancheru, India, 1819 May 2000. International Crops Research Institute for the Semi-Arid Tropics (ICRISAT); 2000.

17. Alberts JF, Engelbrecht Y, Steyn PS, Holzapfel WH, Van Zyl WH. Biological degradation of aflatoxin B 1 by Rhodococcus erythropolis cultures. International journal of food microbiology. 2006; 109(1): 121-6.

18. Taylor JR, Schober TJ, Bean SR. Novel food and non-food uses for sorghum and millets. Journal of Cereal Science. 2006; 44(3): 252-71.

19. Asif M, Rooney LW, Acosta-Sanchez D, Mack CA, Riaz MN. Uses of sorghum grain in glutenfree products. Cereal foods world. 2010; 55(6): 285-91.

20. Marengo M, Bonomi F, Marti A, Pagani MA, 
Elkhalifa AE, Iametti S. Molecular features of fermented and sprouted sorghum flours relate to their suitability as components of enriched gluten-free pasta. LWT-Food Science and Technology. 2015; 63(1):511-8..

21. Oluwafemi F, Kumar M, Bandyopadhyay R, Ogunbanwo T, Ayanwande KB. Biodetoxification of aflatoxin B1 in artificially contaminated maize grains using lactic acid bacteria. Toxin Reviews. 2010; 29(3-4):115-22.

22. El-Nezami H, Kankaanpaa P, Salminen S, Ahokas J. Ability of dairy strains of lactic acid bacteria to bind a common food carcinogen, aflatoxin B 1. Food and Chemical Toxicology. 1998; 36(4): 321-6.

23. Correia I, Nunes A, Guedes S, Barros AS, Delgadillo I. Screening of lactic acid bacteria potentially useful for sorghum fermentation. Journal of cereal science. 2010; 52(1):9-15.

24. Lutfullah G, Hussain A. Studies on contamination level of aflatoxins in some cereals and beans of Pakistan. Food Control. 2012; 23(1): 32-6.

25. Prakash B, Singh P, Kedia A, Singh A, Dubey NK. Efficacy of essential oil combination of Curcuma longa L. and Zingiber officinale Rosc. as a postharvest fungitoxicant, aflatoxin inhibitor and antioxidant agent. Journal of Food Safety. 2012 ; 32(3): 279-88.
26. Rahmani A, Jinap S, Soleimany F. Qualitative and quantitative analysis of mycotoxins. Comprehensive Reviews in Food Science and Food Safety. 2009; 8(3):202-51.

27. Younis YM, Malik KM. TLC and HPLC assay of aflatoxin contamination in Sudanese peanuts and peanut products. Kuwait J Sci Eng. 2003; 30(1): 79-93..

28. Chang HH, De Vries JW, Hobbs WE. Comparative study of two methods for extraction of aflatoxin from peanut meal and peanut butter. Journal-Association of Official Analytical Chemists. 1979; 62(6): 1281-4..

29. Haghighi B, Thorpe C, Pohland AE, Barnett R. Development of a sensitive high-performance liquid chromatographic method for detection of aflatoxins in pistachio nuts. Journal of Chromatography A. 1981; 206(1): 101-8..

30. Mishra HN, Das C. A review on biological control and metabolism of aflatoxin.

31. Peltonen KD, El Nezami HS, Salminen SJ, Ahokas JT. Binding of aflatoxin B1 by probiotic bacteria. Journal of the Science of Food and Agriculture. 2000; 80(13): 1942-5..

32. Lahtinen $\S \mathrm{SJ}$, Haskard CA, Ouwehand $\S \mathrm{AC}$, Salminen § SJ, Ahokas JT. Binding of aflatoxin B1 to cell wall components of Lactobacillus rhamnosus strain GG. Food additives and contaminants. 2004; 21(2): 158-64. 